\title{
Cuerpo y memoria: el ser físico ante la experiencia atroz
}

\author{
Body and memory: the physical being \\ in the face of the atrocious experience
}

GREGORIO SARAVIA MÉNDEZ*

\begin{abstract}
Resumen: El objeto de este trabajo es intentar poner de relieve la naturaleza epistémica, si es que cabe reconocerle alguna, de la experiencia atroz de la tortura y destacar el papel que jugaría el cuerpo de la víctima en la conformación de la memoria. En relación con la primera cuestión, se puede oír hablar a las víctimas de una experiencia de daño y que ello provoque en el oyente -o en el lector- emoción y compasión, pero que allí no pueda extraerse ningún tipo de enseñanza o conocimiento. Sobre la segunda cuestión, está el testimonio y está el silencio. Ambos pueden llegar a conmovernos, pero se verán siempre rodeados de significados ocultos.

Palabras clave: Cuerpo, memoria, tortura, experiencia, víctima, daño.
\end{abstract}

\begin{abstract}
The purpose of this paper is to highlight the epistemic nature, if it is possible to acknowledge any, of the atrocious experience of torture and highlight the role that would play the body of the victim in the formation of memory. In relation to the first issue, you can listen to the victims of an experience of harm and this may cause in the listener -or in the reader- emotion and compassion, but that no kind of learning or knowledge can be drawn from it. On the second issue, there is testimony and there is silence. Both have the potential to move us, but will always be surrounded by hidden meanings.
\end{abstract}

Keywords: Body, memory, torture, experience, victim, harm.

\section{Jean Améry: la parte de la tortura}

Ni siquiera la observación fisiológica ni el registro científico resultan capaces de reunir los tipos de dolor posibles para el ser humano. La sensibilidad física y los umbrales del dolor sólo pueden ser definidos en términos individuales. Por ello, nuestros cuerpos reaccionan de modos muy diversos al dolor y ello resulta aplicable tanto a la hora de medir la capacidad de resistencia como al analizar los factores morales y físicos ${ }^{1}$.

Fecha de recepción: 06/04/2016. Fecha de aceptación: 17/07/2016.

* Universidad Carlos III de Madrid, Profesor Visitante del Departamento de Humanidades: Filosofía, Lenguaje y Literatura, saravia.gregorio@gmail.com, Filosofía Moral, Filosofía Política, Memoria Histórica, Historia de las Ideas. Publicaciones recientes: "Memoria, Justicia Restaurativa y naturaleza de las Reparaciones Simbólicas", Antonio Gómez Ramos \& Cristina Sánchez Muñoz (eds.), Confrontando el mal, Ensayos sobre Violencia, Memoria y Democracia, Ed. Plaza \& Valdés, Madrid, 2016. "Anarchy and Human Rights in the 20th century", Essays on Human Rights, Ed. Aranzadi, 2015, pp. 587-628.

1 Uno de los estudios más completos que existen sobre esta materia es el de Elaine Scarry. Vid. Scarry, E., The body in pain. The making and Unmaking of the World, New York, Oxford University Press, 1987, pp. 3-59. 
De las múltiples causas que pueden provocar dolor, aquí interesa subrayar la que proviene del acto de la tortura. Se trata de un acto específicamente humano, de una de las más brutales manifestaciones del mal ocasionadas por los seres humanos y que se presenta sin límites en el tiempo, en las metodologías y en los fines que persigue. A continuación se ofrecerá una explicación de estas variables y de la manera en que se vinculan.

En relación con el tiempo, la tortura puede extenderse tanto como la propia vida del torturado. En este sentido, hay que tener en cuenta que no siempre el móvil de la tortura responde a la búsqueda de un fin concreto, como puede ser la obtención de información sino al mero ejercicio del sadismo. Por otro lado, los efectos de la tortura en una persona suelen ser indelebles, es decir, el transcurso del tiempo puede cerrar las cicatrices del cuerpo pero no así las que se producen en la estructura psíquica.

Respecto de la ausencia de límites en los métodos de tortura, hay que tener en cuenta que se han practicado desde la antigüedad técnicas tales como la del potro, el torno, el despellejamiento o las mutilaciones hasta llegar a otras, ya en la modernidad, que se encuentran perfeccionadas por el auxilio quirúrgico, farmacológico, químico o tecnológico. Prácticas tales como el empleo de la picana -que opera con descargas eléctricas-, el submarino en sus versiones seca y mojada (consistente en la colocación de una funda plástica en la cabeza del sujeto hasta provocar su ahogamiento o bien la introducción de su cabeza en un tanque con agua y con las piernas suspendidas hacia arriba), la introducción de agujas bajo las uñas, el aislamiento, la privación del sueño, la inyección de sustancias nocivas o las quemaduras con cigarrillos, son tan sólo algunos ejemplos de un catálogo que no ha dejado de expandirse hasta nuestros días.

Es usual que la tortura se refiera a la imposición, de forma intencionada, de dolor o sufrimiento grave, ya sea física o mentalmente, con un propósito específico. Es en relación con éste último criterio en el que la definición de la tortura propuesta resulta insuficiente al menos si atendemos al análisis que de ella ha hecho una de sus más lúcidas víctimas, Jean Améry. Bajo este pseudónimo se conoce al filósofo y escritor vienés Hans Mayer (19121978), figura relevante de la resistencia al nazismo en Bélgica, que fue arrestado y torturado por la Gestapo antes de ser enviado al campo de concentración de Auschwitz.

Veinte años después de Auschwitz, justamente en un ensayo intitulado Más allá de la culpa y la expiación, Améry calificará a la tortura como el acontecimiento más atroz que un ser humano puede experimentar y no llegará a dicha conclusión tomando como base su propio padecimiento sino por razones que, según él, trascienden a la misma.

No se trata de la gravedad de los tormentos lo que suscitó la reflexión de Améry sino los problemas fundamentales de la existencia vinculados con la experiencia atroz de la tortura y el universo concentracionario. Dos cuestiones que para el autor se encontraban estrictamente unidas porque se mostró convencido de que la tortura fue la esencia del Tercer Reich y no un mero elemento accidental. El régimen totalitario bajo las órdenes de Hitler se realizó con plenitud gracias a la tortura. En palabras del autor, los nazis "torturaban con la buena conciencia de su maldad. Martirizaban a sus prisioneros para determinados fines, de vez en cuando exactamente especificados. Pero torturaban sobre todo porque eran verdugos. Se servían de la tortura. Pero aún con mayor fervor actuaban como sus siervos"2.

2 Vid. Améry, J., Más allá de la culpa y la expiación. Tentativas de superación de una víctima de la violencia, Traducción de Enrique Ocaña, Valencia, Pre-textos, 2001, p. 95. 
Los efectos de la tortura empiezan a actuar desde el primer golpe ya que con éste la víctima quedará para siempre marcada, estigmatizada, para siempre. En ese preciso momento la víctima está en condiciones de reconocer que cualquier cosa es posible estando a merced de su verdugo. Diferentes opiniones al respecto parecen coincidir en que con ese primer golpe el detenido pierde su dignidad humana. No obstante, Améry dice ignorar qué significado puede llegar a tener el concepto mismo de «dignidad humana» y se inclina por sostener que "ya con el primer golpe que se le asesta pierde algo que tal vez podríamos denominar provisionalmente confianza en el mundo"3.

La pérdida de confianza en el mundo que produce la tortura implica sobre todo el rebase de una certeza de que "los otros, sobre la base de contratos sociales escritos o no, cuidarán de mí, o mejor dicho, respetarán mi ser físico y, por lo tanto, también metafísico. Las fronteras de mi cuerpo son las fronteras de mi yo. La epidermis me protege del mundo externo: si he de conservar la confianza, sólo puedo sentir sobre la piel aquello que quiero sentir" 4 .

Traspasada dicha frontera a la que se refiere Améry, el torturado queda reducido a su carne, quedan aniquiladas sus esperanzas de defensa y sus expectativas de poder ser rescatado o socorrido. Así es como la tortura supone la aniquilación total de la existencia del torturado, puesto que "el puño del policía, que excluye toda defensa y al que no ataja ninguna mano auxiliadora, acaba con un parte de nuestra vida que jamás vuelve a despertar"5.

Para el sujeto víctima de la tortura la elaboración de la propia vivencia y su incorporación en el conjunto del propio periplo vital se presenta como problemática, cuando no imposible. Esa imposibilidad es la de un encuentro entre el sujeto-víctima y la historia en la que normalmente se integrarían los acontecimientos vividos y que a su vez participan de forma decisiva en la construcción de su personalidad. Esa relación de reciprocidad, ese existir del sujeto por y en la historia es lo que se ha visto brutalmente interrumpido por la tortura.

La negatividad radical del acontecimiento que es la tortura convierte en paradójica la referencia misma a una vivencia, a una positividad factual que el sujeto se representa como propia $^{6}$.

La vida del torturado está marcada por haber tenido un tramo en que no parecía vivir su propia vida. Lo característico de los efectos de la tortura es barrer al sujeto y su personalidad y con ellos la posibilidad para el mismo de construirse en sus vivencias, de incorporarlas al propio relato de la personalidad y en el medio en que éstas se incardinan: el relato más amplio de la historia.

Lo que fracasa además es la elaboración del encuentro entre existencia e historia, o más bien entre la experiencia interior de un acontecimiento extremo que ha interrumpido la propia existencia y la narración colectiva, externa, de ese acontecimiento. La frustración de esta elaboración impide a la vez el encuentro entre ambos relatos, el individual y el colectivo. De alguna manera, el acceso a la propia vivencia que la haría susceptible de ser relatada se trunca en la experiencia misma de la tortura.

3 Vid. Améry, J., Más allá de la culpa y la expiación, op. cit., p. 90.

4 Vid. Améry, J., Más allá de la culpa y la expiación, op. cit., pp. 90 y 91.

5 Vid. Améry, J., Más allá de la culpa y la expiación, op. cit., p. 92.

6 Para un análisis de esta cuestión, vid. Corbí, J. E., Morality, Self-Knowledge and Human Suffering, New York, Routledge, 2012, pp. 45-73. 
Ser torturado es percibir el propio cuerpo de una manera completamente novedosa porque el dolor es la máxima exaltación imaginable de nuestra propia corporalidad. En virtud de ello, "quien ha sido torturado permanece tal. La tortura deja un estigma indeleble, aunque desde un punto de vista clínico no sea reconocible ninguna traza objetiva"7.

Para Jean Améry, el ultraje que supuso la tortura resultó incurable, insuperable. La tortura fue para él "una muerte interminable" ". Su reflexión gira sobre la incapacidad que tiene la experiencia de la tortura para aportar algún tipo de conocimiento que se diferencie de la mera pesadilla. El cuerpo del torturado ha experimentado un sentimiento de extrañeza y estupefacción que no podrá ser nunca compensado. La palabra resulta estéril ante lo que equivale a experimentar que el "otro" puede convertirse en un soberano absoluto que infringe dolor y destruye. El torturador se convierte así en alguien capaz de convertir al torturado en un trozo de mera carne despojado de aquello que podría denominarse alma, espíritu, conciencia o identidad 9 .

La condición existencial de la víctima del nazismo tal como la ha trabajado Améry no deja a lugar a la auto conmiseración ni a la victimización pero sí pone de relieve el valor del resentimiento como un sentimiento que no debe necesariamente confundirse con el deseo de venganza. En su caso personal, este resentimiento parece haberle conducido a la extrema negación de su propia persona: un proceso de derribo interior que finalizó de manera trágica. El 17 de octubre de 1978, Jean Améry se suicidó en la ciudad de Salzburgo.

\section{Reinhart Koselleck: la parte de la memoria}

Si tomamos como referencia a la posición de Améry parece claro que surgen dificultades a la hora de volcar en estructuras gramaticales experiencias de daño y hay que ver cómo el lenguaje, ¿qué lenguaje?, puede ayudar o frenar la comunicación de dichas experiencias. Han existido y existen objeciones a la supuesta fidelidad de los recuerdos a los hechos del pasado y algunos de los testimonios ofrecidos por supervivientes de la barbarie nazi son prueba de ello. En este sentido, existen sospechas sobre la adecuación de la memoria a los hechos pasados e inquietudes respecto a las posibilidades reales de convertir en narración una experiencia de carácter traumático.

7 Vid. Améry, J., Más allá de la culpa y la expiación, op. cit., p. 98.

8 La expresión está tomada de Primo Levi cuando hace referencia a Jean Améry con el que coincidió como prisionero en Auschwitz y con quien debatió años más tarde sobre cuestiones relativas a esta experiencia en común y la manera de tratarla. Vid. Levi, P., Los hundidos y los salvados, Traducción de Pilar Gómez Bedate, Barcelona, Muchnik Editores, 2000, p. 22.

9 “...en el mundo de la tortura, el hombre subsiste sólo en la destrucción del otro. Una ligera presión con la mano provista de un instrumento de suplicio basta para transformar al otro, incluida su cabeza, donde tal vez se conservan las filosofías de Kant, Hegel y las nueve sinfonías completas y El mundo como voluntad y representación, en un puerco que grita estridentemente de terror cuando lo degüellan en el matadero. El torturador mismo puede entonces, cuando ha ejecutado todo, expandiéndose en el cuerpo del prójimo y extinguiendo cuanto le quedaba de espíritu a la víctima, fumarse un cigarrillo o desayunar o, si tienes ganas, ensimismarse en la lectura de El mundo como voluntad y representación”. Vid. Améry, J., Más allá de la culpa y la expiación, op. cit., p. 101. 
Todo discurso sobre la tortura o sobre experiencias atroces tales como las que han definido al exterminio nazi suele hace referencia en algún u otro momento a la categoría de indecible, esto es a la experiencia límite que se resiste a ser puesta en palabras (y por tanto, a ser imaginada y por tanto pensada), ya sea en sentido positivo, para predicar tal adjetivo del tema tratado, o bien para negar o matizar tal carácter. ¿Qué es lo indecible y qué implica? ¿Es posible llegar al núcleo de la experiencia atroz de la tortura o de Auschwitz y extraer de ella algún significado?

Explorar lo indecible de la experiencia atroz lleva ineludiblemente a la figura del testigo, punto originario de todo posible discurso. Por testigo entendemos generalmente el superviviente, quien estuvo en el campo o fue objeto de tortura y sobrevivió. Aquel que padeció la experiencia del exterminio nazi y conservó tras ella la vida. Quien tras ello reingresó, al menos aparentemente, en la sociedad de los hombres donde podría contar lo que vio y vivió. El relato del superviviente se perfila ante nosotros como el relato experiencial de lo atroz, el que procede de la experiencia de seres humanos reales en el mundo extremo de la tortura.

La voz del torturado parece contener lo verdadero de la experiencia, aquello a lo que precisamente querríamos llegar para ver, oír y comprender de qué se trata. Por ello, el testimonio aparece como el espacio privilegiado donde pensar lo indecible, donde la problemática que la atraviesa y la envuelve se manifiesta de una forma más concreta y radical.

El problema más inmediato, que acechaba a muchos prisioneros ya en el campo de concentración y que les acompañó una vez liberados fue el temor a que no les creyeran y en el peor de los casos ni siquiera escuchados por "el mundo de fuera", por quienes nunca vivieron algo similar.

De esta forma, el primer obstáculo para el testimonio del superviviente es el temor a la recepción de su relato por parte de la sociedad a la que se vuelve, ya él subyace la conciencia de la dificultad de decir la propia experiencia.

Siguiendo en parte otros derroteros de reflexión distintos a los descritos, el historiador alemán Reinhart Koselleck también ha incursionado en esta cuestión a partir de varios de sus trabajos. En líneas generales, se puede afirmar que para Koselleck no hay sujeto portador de la memoria colectiva y cualquier formulación de ésta no es más que una excusa para imponer las opiniones de otro o de un grupo. El derecho que tiene la persona en singular a su propio recuerdo, y sin el cual no podría vivir, no puede ser colectivizado.

Subyacente a esta particular posición suya, se halla el fundamento de que el criterio infalible de la verdad es lo experimentado en carne propia. De ahí que sólo los recuerdos primarios, por su evidencia sensible y emocional, serían auténticos.

Entre los trabajos de Koselleck dedicados a la materia, se encuentran dos sugerentes ensayos producidos a finales de los años 90 y comienzos de este nuevo siglo. En ellos, se enfrenta a la mixtificación de la memoria colectiva y a las falsas abstracciones uniformadoras.

En el primero de los que aquí se mencionarán está La discontinuidad del recuerdo ${ }^{10}$, un texto que comienza por el relato de la propia experiencia del autor cuando, como prisionero

10 Originalmente publicado en el Deustche Zeitschrift für Philosophie, vol. 47, n², Berlín, 1999, pp. 213-222, se citará aquí por su versión en español incluida en Koselleck, R., Modernidad, culto a la muerte y memoria nacional, Edición y traducción de Faustino Ocina, Madrid, Centro de Estudios Políticos y Constitucionales, 2011, pp. 39-51. 
de los rusos, fue llevado en mayo de 1945 a Auschwitz. Allí un antiguo interno del campo de concentración de nacionalidad polaca le obligó a pelar patatas de forma más rápida a lo que Koselleck se negó argumentando que ellos, los prisioneros alemanes, no las comerían. El polaco reaccionó queriendo partirle una silla en la cabeza mientras le afirmaba quieres que te rompa el cráneo, vosotros habéis gaseado a millones.

Producto de dicho episodio, Koselleck tomó contacto por vez primera con la magnitud del exterminio practicado por los nazis en los campos del Este y extrajo la tesis de que dicha experiencia primaria resultó intransferible. Si las experiencias resultan intransferibles, toda experiencia secundaria sólo podrá construirse con base en una discontinuidad.

El ejemplo de la generación del 68 en Alemania -compuesta por los nacidos a comienzos de la década de 1940 o incluso al finalizar la guerra- y su intensa crítica moral hacia la generación de sus padres, le sirve a Koselleck para poner de relieve la fragmentariedad, pluralidad y el carácter intransferible de las experiencias primarias. Estos modos de experiencia que se mantienen en espacios fragmentados sólo pueden agregarse a un espacio de recuerdo constatado través de posteriores procesos de condensación, pero éstos serán siempre secundarios y plagados de problemas puesto que "el paso de la experiencia primaria de origen plural y segmentario al recuerdo institucionalizado es un proceso constante, que en cada actualidad fija científicamente y ex post una experiencia (...) que siempre lleva consigo sorpresas para la generación que primariamente la vivió (...) siempre sale a la luz algo nuevo y siempre se sabe algo diferente que antes"11.

Asumiendo que los crímenes del nazismo fueron tan graves que no pueden ser concebibles ni comprensibles, Koselleck no evita abordar tres formas de tratamiento de la cuestión que serían posibles de esgrimir con motivo de la tesis de la discontinuidad. La primera alternativa sería la de recurrir a la ciencia, bien sea la historia, la sociología o la filosofía para explicar qué pasó. Una tarea interminable, según el autor, que siempre puede ser ampliada y por tanto permanece abierta.

La segunda explicación viene constituida por el juicio moral, acerca del exterminio de los judíos, los pueblos eslavos, las etnias sinti y romà, y éste siempre tiene razón. Excepto que uno defienda la ideología nazi, ¿quién podría afirmar que es justo el exterminio de millones de personas inocentes? Ahora bien, la dificultad que supone una explicación de este tipo es que no aporta más que la constatación de fue injusto, fue injusto, fue injusto. Se trata de un tipo de juicio tan correcto como infructuoso.

Por último, es dable hacer referencia a la respuesta religiosa que consistiría en un acto de penitencia perpetuado por la oración que legitima el recuerdo de las víctimas. Se trata de una estrategia en la que quedan fuera los no creyentes y que para Koselleck "no es un asunto del Estado ponerse un traje de religiosidad para celebrar de un modo cuasirreligioso la penitencia por los pecados, la cual, a falta de fe cristiana, ni se puede proveer ni se puede esperar"12.

Ninguna de las tres alternativas de recuerdo resulta satisfactoria para el historiador alemán a la hora de explicar lo que en verdad resulta incomprensible del pasado atroz. Cada

11 Vid. Koselleck, R., "La discontinuidad del recuerdo", en Modernidad, culto a la muerte y memoria nacional, cit., p. 42.

12 Vid. Koselleck, R., "La discontinuidad del recuerdo", cit., p. 47. 
una a su manera sólo puede conducir a la desesperación y quizá sea ésta la que tenga que quedar retenida en el recuerdo o, en palabras de Koselleck, "metafóricamente, cada paso lleva junto a la cámara de gas pero no a su interior"13.

El segundo trabajo de Koselleck que aquí interesa subrayar es Formas y tradiciones de la memoria negativa ${ }^{14}$ que al igual que en el caso anterior se interroga por la posibilidad de recordar crímenes atroces. La tesis aquí defendida resulta ser también la del carácter intransferible que tienen experiencias tales como las que marcaron a millones de víctimas durante el nazismo: el miedo, el hambre, el agotamiento, la falta de protección, la ausencia de cualquier tipo de esperanza de mejora o auxilio. Todas ellas conforman los recuerdos de los afectados que "se derraman como una masa de lava sobre sus cuerpos, de un modo inamovible y permanente"15.

En contraste con este tipo de experiencias, todas las de los que no estuvieron allí, como por ejemplo la de los nacidos en generaciones posteriores, son secundarias.

Así es como pueden identificarse una serie de experiencias de personas que compartieron su sufrimiento en los campos y la conservación de un conjunto de recuerdos que dejaron huella en sus memorias sin intermediarios. No obstante, la enunciación de este fenómeno no puede suponer, según Koselleck, la existencia de una memoria colectiva. Ésta no sería más que una falacia bienintencionada.

Al igual que en el caso de Améry, Koselleck estudia los alcances de este legado negativo pero en su planteamiento adquiere protagonismo la cuestión de la gestión del mismo por parte de la sociedad en su conjunto. En este caso, su estudio se centra en la sociedad alemana y las funciones que al respecto debería cumplir el Estado alemán.

El eje de este trabajo lo constituye tres preguntas básicas: ¿Quién debe ser recordado?; ¿Qué se debe recordar?; ¿Cómo hay que recordar?

Ante una memoria negativa como la alemana, el camino que propone Koselleck consiste en diferenciar las experiencias primarias -protagonizadas por los auténticamente afectados- de la experiencia elaborada y secundaria. Ésta última es la que prevalece en la actualidad y que genera problemas tales como los enunciados a partir de las tres preguntas ya mencionadas.

La memoria alemana, negativamente preformada, sólo puede ser elaborada historiográficamente porque las experiencias de los supervivientes o de los asesinados no pueden ser la de los alemanes actuales.

Respecto de la primera pregunta, el autor sostiene que en su país se debe recordar tanto a los verdugos como a las víctimas. Estrategia que se ha visto obstaculizada por la exclusión mutua que ha tenido lugar sobre todo en términos de representación monumental. Acerca de esta cuestión, Koselleck expone críticamente el caso de la Neue Wache.

En el suelo del interior de la Neue Wache se puede a leer, a los pies de la Pietà de Kollwitz, la inscripción "a las víctimas de la guerra y la tiranía" (Den Opfern von Krieg und Gewaltherrschaft) que es, de acuerdo con Koselleck, equívoca e inapropiada. El término

13 Vid. Koselleck, R., "La discontinuidad del recuerdo", cit., p. 48.

14 Originalmente publicado como "Formen und Traditionen des negativen Gedächtnisses", en Volkhard Knigge y Norbert Frei (eds.), Verbrechen erinnern, Beck, Múnich, 2002, pp. 21-32 vol. 47, se citará aquí por su versión en español incluida en Koselleck, R., Modernidad, culto a la muerte y memoria nacional, cit., pp. 53-63.

15 Vid. Koselleck, R., "Formas y tradiciones de la memoria negativa", cit., p. 55. 
opfern denota tanto el sacrificio voluntario como el hecho de haber sido vencido o muerto. Esto hace que se confundan los soldados alemanes con quienes han sido asesinados por los nazis dando a entender como si todos los alemanes caídos en la guerra hubiesen sido víctimas pasivas del nazismo, al igual que los millones de inocentes asesinados. Una fórmula de estas características encierra una hipocresía, una ambivalencia y un fraude que no hace justicia con el pasado ni con los efectivamente aniquilados.

Tal como se puede apreciar, la construcción cultural de la memoria -aquella que Koselleck designa como resultado de la experiencia elaborada y secundaria- sí que es transmisible a otras generaciones. Las instituciones y entidades, entre ellas desde luego el propio Estado, se forjan una memoria utilizando símbolos, textos, imágenes, lugares, ritos, monumentos y prácticas. Temas como los de la culpa y la vergüenza tienen difícil acceso a la memoria nacional porque cuesta integrarlas en una imagen positiva. En la construcción de la memoria nacional, ésta incorpora elementos enaltecedores y humillantes, pero éstos últimos sólo en el caso de que puedan asociarse con una imagen heroica o martirológica ${ }^{16}$.

La tesis defendida por el autor es que para los alemanes "sólo hay una posibilidad: la autoría y los autores deben ser incluidos en el recuerdo y no sólo las víctimas como tales. Esto nos diferencia de otras naciones. Pues nosotros somos políticamente responsables, y por eso debemos recordar los hechos y los autores y no sólo conmemorar a las víctimas"17.

En última instancia, y dentro del marco de esta pregunta acerca de quién debe ser recordado, surge la dificultad de los grupos de víctimas que tienden a individualizarse entre sí en aras de proteger su identidad. El problema que señala el historiador es que, de esta manera, se perpetúan las categorías que las propias SS habían impuesto a sus prisioneros para clasificarles. Además, sigue abierta, y con polémica, la cuestión de si el duelo por los aniquilados debe seguir haciéndose de forma diferenciada según el grupo de víctimas que se trate: judíos, sinti, romà, homosexuales o, incluso, los propios alemanes. En efecto, Koselleck, al igual que otros intelectuales alemanes como el escritor W. G. Sebald, ha advertido de las deficiencias de una memoria que, como la alemana, ha desterrado del recuerdo a los muertos de su propia nación.

Sobre la segunda pregunta, ¿qué se debe recordar?, la respuesta es más directa y contundente: la verdad. Ahora bien, el propio autor es consciente de que en el ámbito de esta pregunta se aloja el serio problema de pensar lo impensable y representar lo irrepresentable.

Al hilo de lo anterior, aparece la tercera cuestión: ¿cómo se debe recordar? Para esta respuesta Koselleck vuelve a traer a colación, como en el caso del ensayo anterior analizado, las formas del recuerdo moral, el recurso a la ciencia y el culto memorial religioso. Sin embargo, incluye también en esta ocasión la vertiente estética.

16 En este sentido, se trata de una de las dificultades propias de la negatividad de la memoria alemana el limitarse a postular héroes positivos como los de la resistencia al nazismo pero apartando otros muertos suyos. Afirma el autor, "un pequeño monumento a los combatientes de la Primera Guerra Mundial fue decapitado en los años noventa. El libro -eclesiástico- de los difuntos de la Segunda Guerra Mundial fue robado recientemente. No puedo recordar que se haya producido alguna reacción audible. Como si el recuerdo del asesinato de los judíos nos forzara a olvidar a nuestros propios muertos (...) El trato con nuestros muertos que actuaron durante la Segunda Guerra Mundial, que pertenecen a la nación, se halla tan roto que la huida hacia un aparente pacifismo, que derriba y destruye los llamados monumentos militaristas, parece ofrecer una especie de exoneración. Esto es un error". Vid. Koselleck, R., "Formas y tradiciones de la memoria negativa", cit., p. 60.

17 Vid. Koselleck, R., "Formas y tradiciones de la memoria negativa", cit., p. 58. 
En relación con este último elemento, quizá no esté de más recordar que un sello distintivo de las labores de Koselleck como historiador ha sido su interés por la estética de la anamnesis y en particular por todo lo vinculado con los memoriales. A partir de una periodización que separa los monumentos de la Primera Guerra Mundial -en los que predomina un sentido de lo que supuso la muerte de los caídos- de los correspondientes al período histórico posterior, Koselleck sostiene que éstos últimos demandaron un sentido que era muy difícil de encontrar. Después de la Segunda Guerra Mundial, la negatividad de la memoria contemporánea ha devenido en aporía: la estética ha asumido el desafío de representar lo que es irrepresentable, se ha topado con que la cuestión del sentido es un en sí misma un sinsentido. Por ello, es reconocible una temática que caracteriza a muchos monumentos del Holocausto en la que se utilizan "muros que dejan abierta una grieta (...) formas huecas para los desaparecidos (...) masas de piedra acumuladas (...) de este modo los crímenes sólo son indirectamente mostrados"18.

Hacia el final del ensayo, que es objeto aquí de comentario, Koselleck también subraya la relevancia que han adquirido trabajos de ilustración y texto como los de Art Spiegelman en los que sirviéndose de una alusión indirecta, como es la fábula de animales, es capaz de poner al lector en un estado de reflexión que en muchos casos la contemplación de formas directas de representación del horror nazi no ha podido alcanzar.

\section{Alguna conclusión}

Los periplos vitales de Jean Améry y Reinhart Koselleck poco tienen que ver ya que ni siquiera pertenecieron a la misma generación. Sus nacionalidades no fueron las mismas e incluso el primero de ellos por su condición de judío le tocó estar en el bando de las víctimas mientras que al segundo la guerra sólo le afecto incidentalmente. Tampoco fueron comunes sus profesiones. Sin embargo, ambos utilizaron el alemán como lengua de expresión y trabajo, pero quizá lo más destacable es que en ellos hallamos un tipo de reflexión sobre la memoria, el dolor y el cuerpo que tienen sus puntos de confluencia.

Destacaré sólo tres de ellos. El primero es que tanto en el filósofo-escritor como en el historiador-intelectual encontramos una centralidad reconocida a la experiencia atroz como configuradora, de manera radical y permanente, del sujeto. En el estudio que Améry realiza sobre los efectos de la tortura, éstos se resisten a ser elaborados como experiencia formativa al igual que ocurre con las experiencias primarias a las que se refiere Koselleck.

En segundo lugar, podemos mencionar las tesis comunes que han defendido en torno a la memoria negativa como aquella que en la contemporaneidad ha estado en la búsqueda incesante de comprender lo incomprensible. Así como la obra de Améry se mueve dentro del laberinto de lo indecible, como resultado de la atrofia que se produce en el lenguaje por la experiencia de exterminio o de tortura, la de Koselleck busca comprender el conjunto de experiencias extremas dejando en todo momento la puerta abierta a la incorporación de nuevos elementos y siendo consciente de las limitaciones de la historiografía para dar cuenta de las mencionadas experiencias.

18 Vid. Koselleck, R., "Formas y tradiciones de la memoria negativa", cit., p. 58. 
En tercer lugar, y siguiendo coordenadas que nos proveen ambos autores, podemos afirmar que lo vivido en primera persona tiene un derecho de veto frente a las memorias de instituciones y entidades que forjan una memoria utilizando diferentes estrategias y materiales propios de una construcción cultural que busca ser transmisible a otras generaciones pero al precio de tergiversar los hechos. De ahí que al historiador le corresponda asumir la supervisión de las diversas instancias transmisoras de los recuerdos secundarios, valorando la importancia de la experiencia directa del daño.

\section{Bibliografía}

Améry, Jean (2001): Más allá de la culpa y la expiación. Tentativas de superación de una víctima de la violencia, Traducción de Enrique Ocaña, Pre-textos, Valencia.

Corbí, Josep E. (2012): Morality, Self-Knowledge and Human Suffering, Routledge, New York, pp. 45-73.

Koselleck, Reinhart (2011): Modernidad, culto a la muerte y memoria nacional, Edición y traducción de Faustino Ocina, Centro de Estudios Políticos y Constitucionales, Madrid.

Levi, Primo (2000): Los hundidos y los salvados, Traducción de Pilar Gómez Bedate, Muchnik Editores, Barcelona.

Scarry, Elaine (1987): The body in pain. The making and Unmaking of the World, Oxford University Press, New York, pp. 3-59. 